\title{
Adhesive Bioactive Coatings Inspired by Sea Life
}

\author{
Sónia J. Rego, ${ }^{\dagger,+, \S}$ Ana C. Vale, ${ }^{\dagger, \ddagger \S}$ Gisela M. Luz, ${ }^{\dagger,+}$ João F. Mano, ${ }^{\dagger,+}$ and Natália M. Alves ${ }^{*}, \dagger,+$
}

${ }^{\dagger} 3$ B’s Research Group - Biomaterials, Biodegradables and Biomimetics, University of Minho, Headquarters of the European Institute of Excellence on Tissue Engineering and Regenerative Medicine, AvePark, 4805-017 Barco GMR, Portugal

${ }^{\ddagger}$ ICVS/3B’s PT Government Associate Laboratory, Braga/Guimarães, Portugal

\section{Supporting Information}

ABSTRACT: Inspired by nature, in particular by the marine mussels adhesive proteins (MAPs) and by the tough brick-andmortar nacre-like structure, novel multilayered films are prepared in the present work. Organic-inorganic multilayered films, with an architecture similar to nacre based on bioactive glass nanoparticles (BG), chitosan, and hyaluronic acid modified with catechol groups, which are the main components responsible for the outstanding adhesion in MAPs, are developed for the first time. The biomimetic conjugate is prepared by carbodiimide chemistry and analyzed by ultraviolet-visible spectrophotometry. The buildup of the multilayered films is monitored with a quartz crystal microbalance with dissipation monitoring, and their topography is charac-

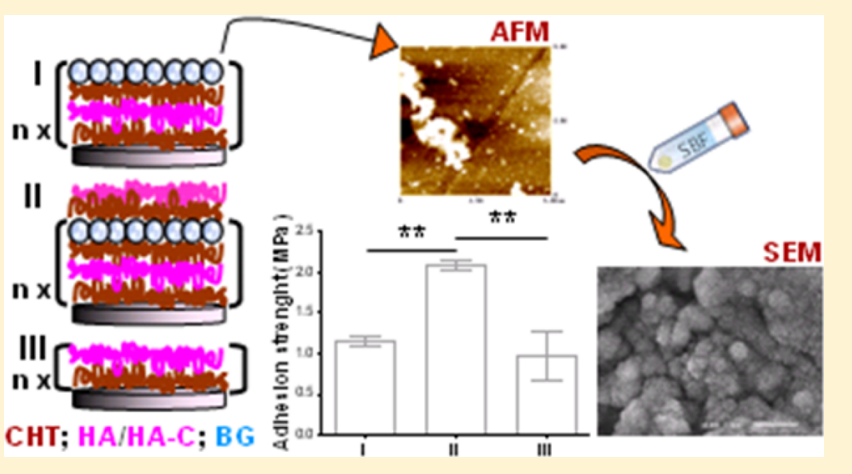
terized by atomic force microscopy. The mechanical properties reveal that the films containing catechol groups and BG present an enhanced adhesion. Moreover, the bioactivity of the films upon immersion in a simulated body fluid solution is evaluated by scanning electron microscopy coupled with energy dispersive X-ray spectroscopy, Fourier transform infrared spectroscopy, and $\mathrm{X}$-ray diffraction. It was found that the constructed films promote the formation of bonelike apatite in vitro. Such multifunctional mussel inspired LbL films, which combine enhanced adhesion and bioactivity, could be potentially used as coatings of a variety of implants for orthopedic applications.

\section{INTRODUCTION}

Nature presents several examples of functional materials and has inspired scientists and engineers in mimicking nature to produce high-performing and environmentally friendly materials. Two of the best studied examples are the rough nacre structure and the water resistant adhesive proteins secreted by marine mussels. ${ }^{1,2}$

The development of adhesives capable to improve cell attachment, proliferation and adhesion to tissues has been a challenge in biomedical applications. ${ }^{3}$ Marine mussels secrete adhesive proteins (MAPs), ${ }^{4,5}$ which contain considerable concentrations of an amino acid designated as 3,4-dihydroxyL-phenylalanine (DOPA). ${ }^{6,7}$ A particular feature of DOPA and its analogue dopamine is the orthodihydroxyphenyl functional group, capable of creating strong bonds in aqueous environments with numerous inorganic or organic surfaces. ${ }^{7-14}$ In this sense, different techniques, such as layer-by-layer (LbL), have been applied in different studies where adhesives are trying to mimic the adhesion properties of marine mussels. ${ }^{3,5,8}$

Regarding biomimetic materials, the hierarchical structure of nacre has also drawn much attention to the scientific community. Nacre is a natural laminate composite made of microscopic aragonite polygonal tablets between thin sheets of organic matrix, which is formed by a protein-polysaccharide ( $\beta$-chitin, silklike proteins, and acidic glycoproteins rich in aspartic acid). ${ }^{15,16}$ On this brick-and-mortar structure of the nacre, the microscopic bricks (aragonite tablets) are arranged in a specific three-dimensional fashion representing $95 \%$ in volume, while the protein-polysaccharide layers act as mortar, providing cohesion and energy dissipation. ${ }^{16}$ Due to this staggered microstructure, that evidence a controlled sliding of the mineral tablets, nacre combines stiffness, remarkable strength, and high toughness. ${ }^{16-19}$ Inspired by the excellent mechanical properties evidenced by the inorganic-organic nanostructure of nacre, the $\mathrm{LbL}$ technique has been widely explored for the construction of organic-inorganic nanostructured thin films. ${ }^{19-22}$

In the context of bioactive materials, it is well-known that some ceramics and glasses have the ability of binding to bone without the formation of a fibrous capsule, with bioactive glass being the most well-known example. ${ }^{23,24}$ Upon implantation, when in contact with bone, bioactive materials bond chemically with bone by forming a hydroxyapatite layer identical to the bone mineral phase. ${ }^{24}$ Bioactive glass nanoparticles, besides their bioactivity, present a potential effect on improving osteoconductivity, cell proliferation, differentiation, and vascularization. $^{19,25-27}$

Received: October 2, 2015

Revised: December 9, 2015

Published: December 9, 2015 
So, inspired by nature, in particular by the inorganic-organic composites nacre-like and by mussels adhesive proteins (MAPs), new multifunctional mussel inspired LbL films were developed by alternate combination of bioactive nanoparticles with biopolymeric layers bearing catechol groups. Such LbL films were prepared by combining dopamine-modified hyaluronic acid (HA-C), chitosan ( $\mathrm{CHT})$, and bioactive glass nanoparticles (BG), where catechol groups present in dopamine were used as a "bioglue" and bioactive nanoparticles layers as "bricks".

The in vitro formation of hydroxyapatite on the films surfaces is a strong indication that the mineralization will also occur in vivo creating a bonelike scaffold pathway that will allow for osteoconductivity, while being able to chemically connect with the host tissue. Several studies showed already that cell response is improved in the presence of the ions released from the BG nanoparticles. ${ }^{19,28,29}$ Therefore, we believe that these films could be used as coatings for a variety of implants for orthopedic applications, in order to improve both cell response and osteoconductivity in a simple and versatile way, avoiding the typical use of cements.

\section{MATERIALS AND METHODS}

Materials Used. Medium molecular weight chitosan $(\mathrm{Mw}=770$ $\mathrm{kDa}$, degree of deacetylation of $79 \%$ ), hyaluronic acid sodium salt from Streptococcus equi $(\mathrm{Mw}=595 \mathrm{kDa})$, dopamine hydrochloride, $\mathrm{N}$ - $(3$ (dimethylamino)propyl)- $\mathrm{N}^{\prime}$-ethylcarbodiimide hydrochloride, dialysis tubing cellulose membrane (avg flat width $33 \mathrm{~mm}$ ), citric acid monohydrate, ammonium phosphate dibasic, calcium nitrate tetrahydrate, ethanol absolute, ammonium hydroxide solution (maximum of $\mathrm{NH}_{3}$ ), polyethylene glycol, sodium chloride, sodium of hydrogen carbonate, potassium chloride, disodium hydrogen phosphate trihydrate, magnesium chloride hexahydrate, calcium chloride, sodium sulfate, tris(hydroxymethyl)aminomethane, hypodrochloric acid, polyethylenimine, and sodium azide were purchased from Sigma-Aldrich. Tetraethyl orthosilicate was purchased from Merck Chemicals.

Except the chitosan, all reagents were used without further purification. Chitosan was previously purified by recrystallization following the procedure described in detail by Couto and coworkers. ${ }^{19}$

Synthesis of the Hyaluronic Acid-Dopamine Conjugate (HAC). Based on the procedure proposed by Lee and co-workers ${ }^{30}$ with some modifications, HA-C was prepared by carbodiimide chemistry using $N$-(3-(dimethylamino)propyl)- $N^{\prime}$-ethylcarbodiimide hydrochloride (EDC) as an activation agent of the carboxyl groups. Hyaluronic acid (HA, $1 \mathrm{~g}$ ) was dissolved in phosphate buffered saline solution (PBS, $100 \mathrm{~mL}$ ), and the $\mathrm{pH}$ was adjusted to 5.5 using hydrochloric acid $(\mathrm{HCl})$ or sodium hydroxide $(\mathrm{NaOH})$ aqueous solutions $(0.5 \mathrm{M})$ under magnetic stirring. The solution was purged with nitrogen for 30 $\mathrm{min}$ to limit the oxygen interaction with the solution. HA-C conjugate was synthesized by addition of $\mathrm{N}$-(3-(dimethylamino)propyl)- $\mathrm{N}^{\prime}$ ethylcarbodiimide hydrochloride (EDC, $338 \mathrm{mg}$ ) and dopamine (DN, $474 \mathrm{mg}$ ) to the previous solution with an acidic $\mathrm{pH}$ of 5.5 and at $4{ }^{\circ} \mathrm{C}$, for $2 \mathrm{~h}$. The unreacted chemicals were removed by dialysis using distillate water for a week, and then the HA-C conjugate was lyophilized for 4 days. As dopamine is sensitive to air and light, all of the reaction was performed at $4{ }^{\circ} \mathrm{C}$ and protected from light, in order to avoid the oxidation of dopamine. In addition, the produced HA-C was also stored at $4{ }^{\circ} \mathrm{C}$ and protected from the light.

Bioactive Glass Nanoparticles (BG) Production. Bioactive glass nanoparticles were prepared using the protocol proposed in two previous works, ${ }^{19,21}$ which is a sol-gel method already optimized to obtain the ternary system of $\mathrm{BG}\left(\mathrm{SiO}_{2} / \mathrm{CaO} / \mathrm{P}_{2} \mathrm{O}_{5}(\mathrm{~mol} \%)=\right.$ 50:45:5).

First, at room temperature, calcium nitrate tetrahydrate $(7.639 \mathrm{~g})$ was dissolved in deionized water $(120 \mathrm{~mL})$. Then, tetraethyl orthosilicate $(9.8353 \mathrm{~mL})$ and ethanol absolute $(60 \mathrm{~mL})$ was added to the calcium nitrate solution. The $\mathrm{pH}$ was adjusted to 2 with a citric acid solution $(30 \mathrm{~mL}, 10 \%)$, and the reaction was left to agitation for 3 $\mathrm{h}$ to produce solution A. Solution B was also prepared with ammonium phosphate dibasic (1.078 g) dissolved in deionized water, and ammonium hydroxide solution was used to adjust the $\mathrm{pH}$ to 11.5. Afterward, solution A was added drop-by-drop to solution B, and at the same time the $\mathrm{pH}$ of solution $\mathrm{B}$ was maintained at 11.5 using ammonium hydroxide solution. This mixture was left to dissolve under magnetic stirring during $48 \mathrm{~h}$, and then a resting period of $24 \mathrm{~h}$ followed. The resulting precipitate was washed three times with distilled water to develop a slurry gel. Then, this BG precipitate was stored for $24 \mathrm{~h}$ at $-80^{\circ} \mathrm{C}$, in order to be subsequently freeze dried for 7 days. Finally, to obtain white bioactive glass nanoparticles with improved bioactivity, the white gel powder was calcinated at $700{ }^{\circ} \mathrm{C}$ for $5 \mathrm{~h}^{28}$ This procedure is already optimized, and the $\zeta$ potential of the obtained BG had been evaluated in previous work using the laser Doppler velocimetry mode of the Nano-ZS instrument (Malvern). ${ }^{19}$

Ultraviolet-Visible (UV-Vis) Spectrophotometry Characterization of HA-C Conjugate. Ultraviolet-visible spectrophotometry was used to confirm if the modification of hyaluronic acid with catechol groups was successful, measuring the absorbance between 200 and $600 \mathrm{~nm}$. A solution of HA-C conjugate of $0.5 \mathrm{mg} \cdot \mathrm{mL}^{-1}$ in $0.15 \mathrm{M}$ sodium chloride was prepared for the UV-vis study. The equipment used was the UV-visible spectrophotometer (Shimadzu UV-1601) using $1 \mathrm{~cm}$ quartz cells.

QCM-D Analysis of the Multifunctional LbL Films Production. The formation of films with low number of layers was followed in situ by quartz crystal microbalance with dissipation monitoring (QCM-D; Q-Sense, E4 system) in order to find the suitable conditions for the LbL assembly. QCM-D is a sensitive sensor of mass variation that also measures the changes in the resonant frequency and the energy dissipation of the adsorbed layer at the crystal surface. In addition, the energy dissipation allows characterization of its viscoelastic properties. ${ }^{3}$

LbL coatings were produced using fresh solutions prepared in 0.15 $\mathrm{M}$ sodium chloride: $0.5 \mathrm{mg} \mathrm{mL}^{-1}$ CHT solution with $1 \%(\mathrm{v} / \mathrm{v})$ of acetic acid, $0.5 \mathrm{mg} \mathrm{mL}^{-1} \mathrm{HA}-\mathrm{C}$ solution, and also $2.5 \mathrm{mg} \mathrm{mL}^{-1}$ BG suspension. The multilayered systems were assembled at $\mathrm{pH}$ of 5.5 adjusted with $\mathrm{NaOH}$ or $\mathrm{HCl}$ solution. BG and $\mathrm{HA}-\mathrm{C}$ were used as polyanion, while CHT was used as polycation. Bioactive glass nanoparticles produced were alternately combined with chitosan and the conjugate (HA-C) to produce bioactive and adhesive multilayered films.

The basic process consists of sequential adsorptions onto the QCMD crystals, with $[\mathrm{CHT} / \mathrm{HA}-\mathrm{C} / \mathrm{CHT} / \mathrm{BG}]_{6}$ and control films with $[\mathrm{CHT} / \mathrm{HA} / \mathrm{CHT} / \mathrm{BG}]_{6}$, since it was previously known that the multilayered films formed with [CHT/HA-C] were possible. ${ }^{3}$ The QCM-D crystals were excited at 15, 25, 35, 45, and $55 \mathrm{MHz}$ corresponding to the third, fifth, seventh, ninth, and eleventh overtones.

Some strategies were adopted to avoid nanoparticles agglomeration, such as using ultrasonication, between 5 and $10 \mathrm{~min}$, to better disperse BG. The system started with $0.15 \mathrm{M} \mathrm{NaCl}$ solution (buffer baseline), and then the polyelectrolyte solutions were injected into the cell during $10 \mathrm{~min}$ (for CHT and HA-C) or $20 \mathrm{~min}$ (for BG) at a flow rate of $50 \mu \mathrm{L} \cdot \mathrm{min}^{-1}$, using a peristaltic pump. The first injected polyelectrolyte was $\mathrm{CHT}$, followed by a washing step of $5 \mathrm{~min}$ after each polyelectrolyte deposition.

Films with 12 bilayers were produced to verify if the film buildup with the proposed materials was possible. The $\mathrm{pH}$ of the solution was adjusted to 5.5 with $\mathrm{NaOH}$ or $\mathrm{HCl}$ solutions, and all experiments were performed at room temperature $\left(25^{\circ} \mathrm{C}\right)$.

Film viscoelastic properties were modeled using the Voigt based model represented by a spring and dashpot in parallel, ${ }^{31}$ and it was assumed a fluid density of $1000 \mathrm{~kg} \cdot \mathrm{m}^{3}$, a layer density of $1200 \mathrm{~kg} \cdot \mathrm{m}^{3}$, and a fluid viscosity of $0.001 \mathrm{~Pa} \cdot \mathrm{s}$. The QTools software (Q-Sense) was used for the data analysis according to the Voigt model, and then the thickness was calculated by fitting the changes in resonance frequency and dissipation using a simplex algorithm to find the 
minimum in the sum of squares of the scaled errors between the experimental and model $\Delta f$ and $\Delta D$ values. ${ }^{31,32}$

Production of the Multifunctional LbL Films. The film deposition through the LbL technique onto a glass plaque was performed using an automatic dip coating equipment. The substrates were dipped alternately in the polyelectrolyte solutions in order to produce films with 10 or 11 layers, depending on the film configuration, and to be able to compare the results with the ones obtained in a previous work. ${ }^{3}$ Polyelectrolytes were deposited using exactly the same conditions described for the QCM experiments.

Distinct films were developed: the multifunctional films containing $[\mathrm{CHT} / \mathrm{HA}-\mathrm{C} / \mathrm{CHT} / \mathrm{BG}]_{5}+\mathrm{CHT} / \mathrm{HA}-\mathrm{C},[\mathrm{CHT} / \mathrm{HA}-\mathrm{C} / \mathrm{CHT} / \mathrm{BG}]_{5}$, and respective controls $[\mathrm{CHT} / \mathrm{HA} / \mathrm{CHT} / \mathrm{BG}]_{5}+\mathrm{CHT} / \mathrm{HA}$ and $[\mathrm{CHT} / \mathrm{HA} / \mathrm{CHT} / \mathrm{BG}]_{5}$. An initial precursor poly(ethylenimine), PEI, layer was used by immersing the substrate in a $5 \mathrm{mg} \cdot \mathrm{mL}^{-1}$ PEI saline solution $(0.15 \mathrm{M} \mathrm{NaCl})$ during $20 \mathrm{~min}$, before the multilayered deposition. These configurations were used to study the effect of the conjugate (HA-C) or the bioactive glass nanoparticles in the last layer on both adhesive and bioactivity properties.

Surface Characterization. Atomic force microscopy imaging was performed to study the topography of the multifunctional films, in air and at room temperature, using a Multimode microscopy controlled by the NanoScope III from Digital Instruments system. Three images were obtained in different regions of each scanned sample $(5 \times 5$ $\mu \mathrm{m}^{2}$ ), using a antimony (n) doped Si probe (TESP probe, Bruker) with a resonance frequency of $320 \mathrm{kHz}$ and a spring constant of $42 \mathrm{~N} /$ $\mathrm{m}$, in PeakForce Tapping mode at a frequency of $1 \mathrm{~Hz}$ and containing 512 scanned lines per image.

Adhesion Tests. In order to measure the adhesive strength of the produced films onto the surface of glass plaques, adhesive tests were performed using a universal mechanical testing machine (INSTRON, model 5540), with a load cell of $1 \mathrm{kN}$, and following an adaptation of the lap shear test described in the ASTM D1002 standard procedure. Immediately after the LbL deposition, pairs of glass plaques were put in contact with an overlapping area $\left(A_{0}\right)$ of $15 \times 20 \mathrm{~mm}^{2}$. Then, to promote a better adhesion, the glass plaques in contact were clamped tightly and maintained at $37^{\circ} \mathrm{C}$ overnight.

For the adhesion experiments, each extremity of bonded samples was placed in the grips of the mechanical testing machine, and an axial tensile force was applied at a constant cross-head speed $\left(5 \mathrm{~mm} \cdot \mathrm{min}^{-1}\right)$ in the upper substrate edge and it pulled until the detachment of the glass plaques. The testing machine was operated by materials testing software (Bluehill2, INSTRON Corporation), and stress-strain curves were calculated for each sample from the load applied $(F)$ and displacement $(\Delta L)$ data acquired, taking into consideration the initial length $\left(L_{0}\right)$ and the overlapped area $\left(A_{0}\right)$.

From the conventional stress-strain curves, two mechanical parameters were calculated: the maximum tensile stress, $\sigma_{\text {TENSmax }}$ which is the maximum stress that the film can support without detachment, and the maximum tensile strain, $\varepsilon_{\text {TENSmax }}$ which corresponds to the strain value achieved for the maximum tensile stress.

In Vitro Bioactivity Studies. Standard in vitro bioactivity studies were conducted by immersing the multilayered films in a simulated body fluid, SBF, solution since it simulates the concentration of ionic species presents in human blood plasma. The SBF solution was prepared by following the Kokubo and Takadama protocol, ${ }^{33}$ and its $\mathrm{pH}$ was adjusted to 7.4. Each coated glass piece was immersed during 7 days in $25 \mathrm{~mL}$ of SBF solution at $37^{\circ} \mathrm{C}$. After removing from SBF solution, these glass pieces were cleaned with ultrapure water and dried at room temperature.

The formation of apatite was characterized by a surface analysis using distinct techniques, such as scanning electron microscopy coupled with energy dispersive X-ray spectroscopy, Fourier transform infrared spectroscopy, and X-ray diffraction.

In Vitro Biocompatibility. L929 mouse fibroblast line (European collection of cell culture, ECACC, U.K.) was used to test the in vitro biocompatibility of the membranes.

Direct contact tests were carried out. The samples were previously sterilized by immersion in $70 \%(\mathrm{v} / \mathrm{v})$ ethanol for $2 \mathrm{~h}$ and then washed twice with sterile phosphate buffered saline (PBS). The cells were seeded in the samples $(n=3)$ at a density of 10000 cells/sample and nourished with Dulbecco's modified minimum essential medium (DMEM) supplemented with $10 \%$ fetal bovine serum (FBS) and $1 \%$ antibiotic. The cultures were incubated at $37^{\circ} \mathrm{C}$. After each time point (1, 3, and 7 days of culture), MTS (3-(4,5-dimethylthiazol-2-yl)-5-(3carboxymethoxyphenyl)-2-(4-sulfophenyl)-2H-tetrazolium) test was performed to determine the cytotoxicity of the membranes. The relative cell viability (\%) was determined and compared with tissue culture polystyrene (TCPS) (positive control of cell viability). Latex was used as negative control of cellular viability. Metabolic activity of cells can be monitored using the tetrazolium compound (MTS), since it is chemically reduced by the mitochondria of the cells into formazan. For this assay, an MTS solution was prepared by using a 1:5 ratio of MTS reagent and D-MEM culture medium without phenol red or FBS, followed by a $3 \mathrm{~h}$ incubation period at $37^{\circ} \mathrm{C}$. All cytotoxicity tests were conducted by using three replicates. Finally, the optical density (OD) was read at $490 \mathrm{~nm}$ on a multiwell microplate reader (Synergy HT, Bio-Tek Instruments).

\section{RESULTS AND DISCUSSION}

Synthesis of the HA-C Conjugate. The HA-C was synthesized by conjugation of dopamine using carbodiimide chemistry and characterized by ultraviolet-visible (UV-vis) spectrophotometry to confirm if the modification of HA with catechol groups was successful. Figure 1 illustrates that the HA-

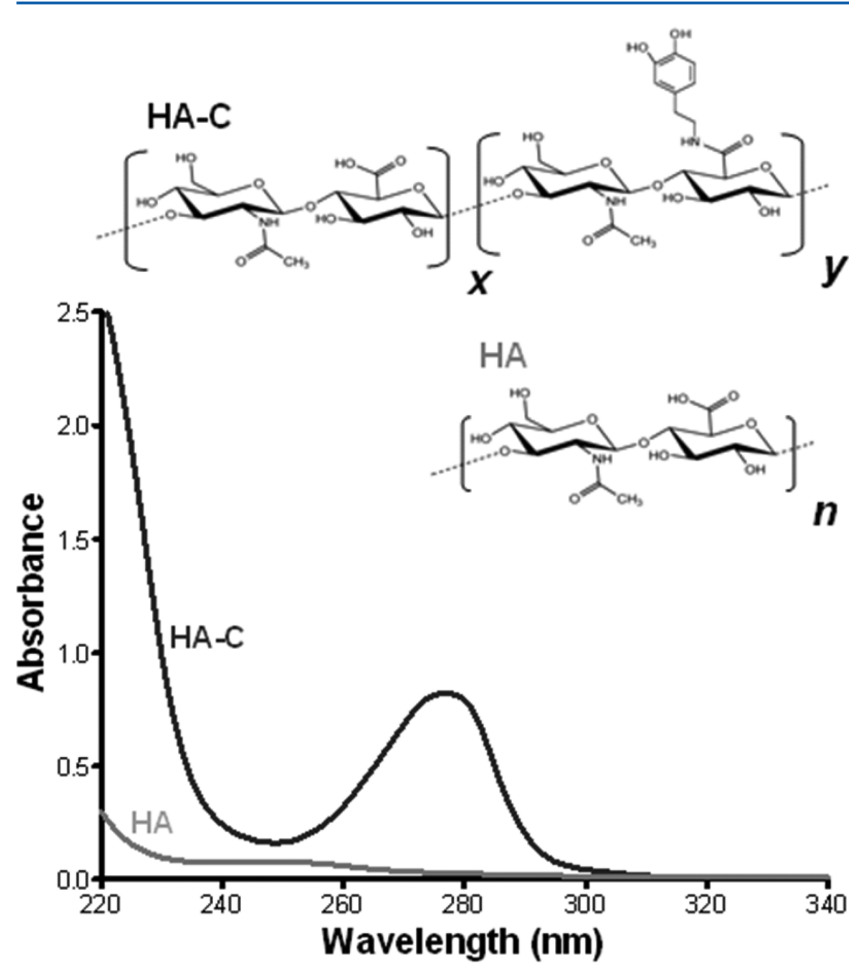

Figure 1. UV-vis spectra of the synthesized HA-C and the HA as the control with their respective structural formulas.

C spectrum presents a maximum absorbance wavelength at approximately $280 \mathrm{~nm}$, in opposition with the HA spectrum that does not present any peak. These results confirm the presence of dopamine in the produced HA-C with the identification of its characteristic peak in the UV-vis spectrum. ${ }^{3,34}$ Moreover, the lack of additional peaks at wavelengths after $300 \mathrm{~nm}$ shows that oxidation of the synthesized conjugate did not occur. ${ }^{3,13}$ The degree of dopamine substitution of the HA-C conjugate was also 

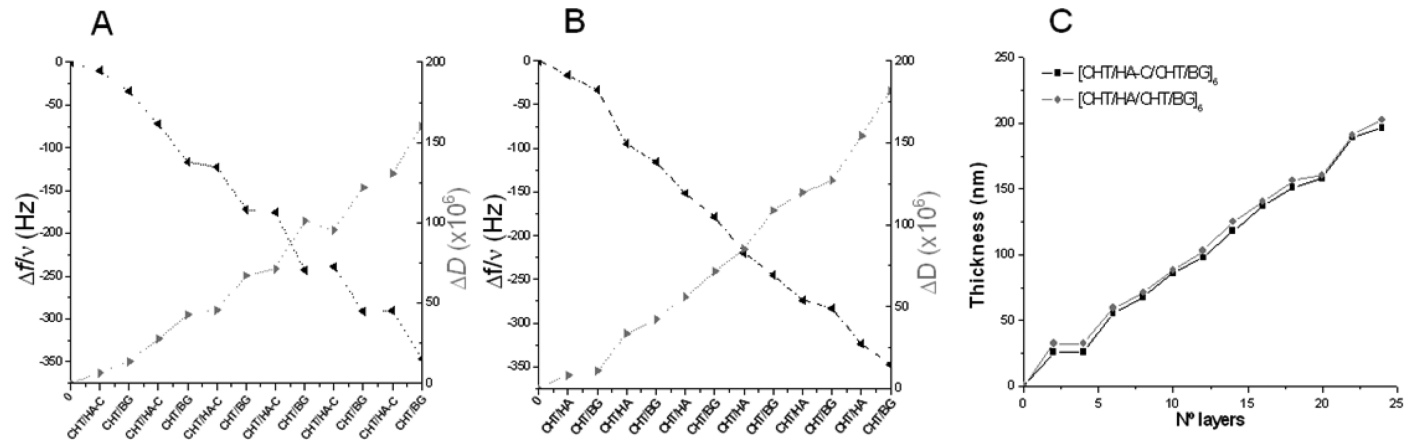

Figure 2. QCM-D with normalized frequency $(\Delta f / v)$ and dissipation $(\Delta D)$ variations at the seventh overtone as a function of the deposition bilayer for $(\mathrm{A})$ adhesive condition $[\mathrm{CHT} / \mathrm{HA}-\mathrm{C} / \mathrm{CHT} / \mathrm{BG}]_{6}$ and $(\mathrm{B})$ respective control $[\mathrm{CHT} / \mathrm{HA} / \mathrm{CHT} / \mathrm{BG}]_{6}$. (C) Cumulative thickness evolution of $[\mathrm{CHT} / \mathrm{HA}-\mathrm{C} / \mathrm{CHT} / \mathrm{BG}]_{6}$ and $[\mathrm{CHT} / \mathrm{HA} / \mathrm{CHT} / \mathrm{BG}]_{6}$ polymeric films as a function of the number of deposition layers. Thickness measurements were estimated using a Voigt viscoelastic model.

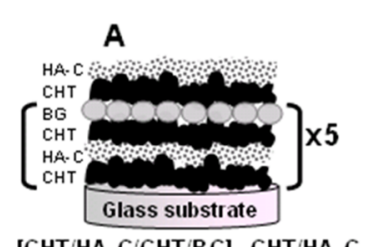

$\left[\mathrm{CHT} / \mathrm{HA}-\mathrm{C} / \mathrm{CHT} / \mathrm{B} \mathrm{G}_{5}-\mathrm{CHT} / \mathrm{HA}-\mathrm{C}\right.$

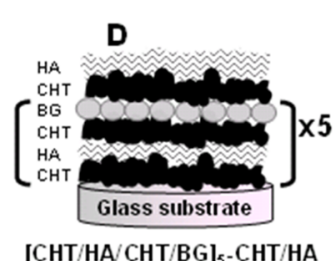

\section{B}

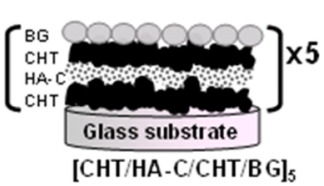

E

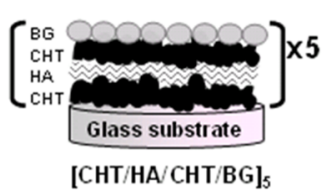

C

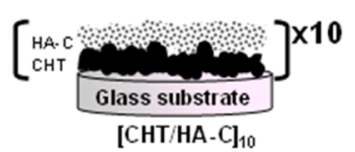

$\mathbf{F}$

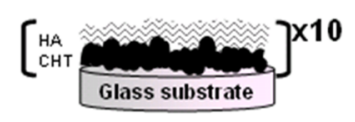

$[\mathrm{CHT} / \mathrm{HA}]_{10}$

Figure 3. Schematic of the multilayer film configuration of (A) condition 1, $[\mathrm{CHT} / \mathrm{HA}-\mathrm{C} / \mathrm{CHT} / \mathrm{BG}]_{5}-\mathrm{CHT} / \mathrm{HA}-\mathrm{C}$; (B) condition 2, [CHT/HA$\mathrm{C} / \mathrm{CHT} / \mathrm{BG}]_{5} ;$ (C) condition 3, [CHT/HA-C $]_{10}$; (D) control 1, [CHT/HA/CHT/BG $]_{5}-\mathrm{CHT} / \mathrm{HA} ;(\mathrm{E})$ control 2, $[\mathrm{CHT} / \mathrm{HA} / \mathrm{CHT} / \mathrm{BG}]_{5}$; and (F) control 3, $[\mathrm{CHT} / \mathrm{HA}]_{10}$.

determined by UV-vis spectrophotometry, and it was nearly $11 \%$, which was in agreement with the value found in previous works. ${ }^{3,34,35}$

QCM-D Analysis of the Multifunctional LbL Films Production. The buildup of multifunctional LbL films with $\mathrm{CHT}, \mathrm{HA}-\mathrm{C} / \mathrm{HA}$, and BG was monitored using quartz crystal microbalance with dissipation monitoring (QCM-D). The results for the construction of $[\mathrm{CHT} / \mathrm{HA}-\mathrm{C} / \mathrm{CHT} / \mathrm{BG}]_{6}$ polymeric films and a control film without dopamine, [CHT/ $\mathrm{HA} / \mathrm{CHT} / \mathrm{BG}]_{6}$, are presented in Figure 2. Figure 2A,B shows the normalized frequency $(\Delta f / v)$ and energy dissipation $(\Delta D)$ variations at the seventh overtone as a function of the deposited layers. A frequency decrease is observed after each layer, proving the adsorption of the corresponding polyelectrolyte, and so the construction of the multilayered film. Also, each adsorbed layer is accompanied by an increase of dissipation, which proves that the film is not rigid, representing the characteristic viscoelastic behavior of polymeric materials. As dissipation increases, the overtones are also becoming separated, which is a characteristic behavior of a soft and hydrated system. It can be seen that there are no substantial differences in normalized frequency values between the two multilayers; however, the lower dissipation variation of the control films indicates that they should be probably thinner, more rigid, and less water-rich. ${ }^{3}$ The use of hydrophilic polymeric materials in the buildup of these multifunctional films is the main reason for the increase in dissipation values, and for the improvement of the assembly viscoelastic properties. Upon assembling with inorganic nanoparticles, nanocomposite films with enhanced mechanical properties were obtained, due to the alternate deposition of BG aggregates with viscoelastic polyssacharides. Similarly with the lamellar structure of nacre, the constructed coatings presented a nanosized inorganic phase (BG nanoparticles agglomerates) arranged in an matrix of biopolymer, which presents inelastic deformations that provides the stress redistribution around strain concentration sites and the elimination of stress concentration. ${ }^{18}$ In addition, as the aragonite platelets from the structure of nacre, the BG nanoparticle aggregations should be ductile, since they can deflect the crack to a direction with an unfavorable stress state and their rotation and deformation are helped by the biopolymer spacing between these nanograins, contributing to better energy dissipation. ${ }^{18,36}$

Figure 2C represents the cumulative thickness evolution of $[\mathrm{CHT} / \mathrm{HA}-\mathrm{C} / \mathrm{CHT} / \mathrm{BG}]_{6}$ and $[\mathrm{CHT} / \mathrm{HA} / \mathrm{CHT} / \mathrm{BG}]_{6}$ films as a function of the number of deposited layers, which evidence that both films present a similar increase of thickness. Some authors have reported an exponential growth of multilayered films based on chitosan and hyaluronic acid, ${ }^{3,37}$ which was not observed in the films developed in this work, probably due to the presence of the bioactive nanoparticles. The final thickness obtained for the control film is approximately $202 \mathrm{~nm}$, which is somewhat higher than for $[\mathrm{CHT} / \mathrm{HA}-\mathrm{C} / \mathrm{CHT} / \mathrm{BG}]_{6}(196$ $\mathrm{nm})$, as expected due to the dissipation variation already 

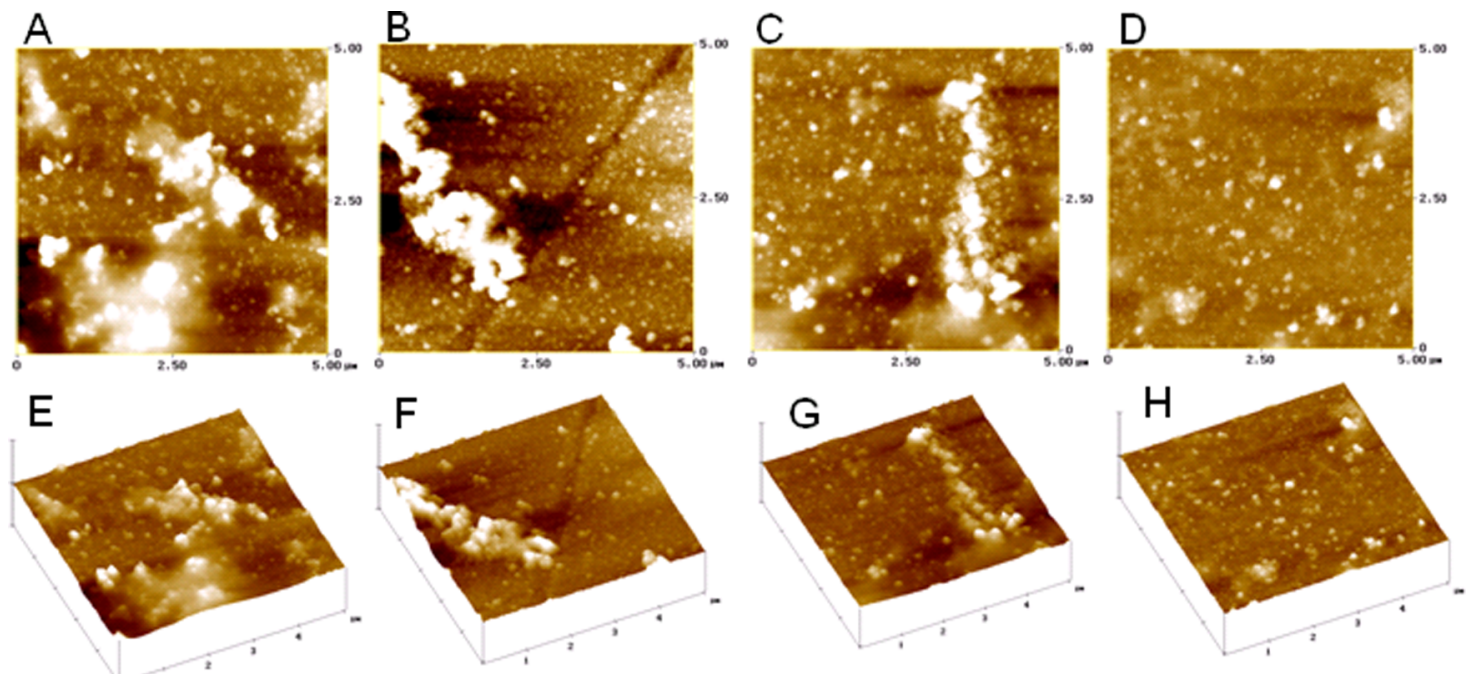

\begin{tabular}{|l|c|c|c|c|}
\cline { 2 - 5 } \multicolumn{1}{c|}{} & Condition 1 & Condition 2 & Control 1 & Control 2 \\
\hline $\mathrm{Rq}(\mathrm{nm})$ & $35.4 \pm 5.2$ & $34.3 \pm 11.0$ & $34.5 \pm 16.4$ & $15.9 \pm 3.9$ \\
\hline $\operatorname{Ra}(\mathrm{nm})$ & $27.5 \pm 3.7$ & $22.2 \pm 7.5$ & $25.4 \pm 13.3$ & $10.3 \pm 2.6$ \\
\hline
\end{tabular}

Figure 4. $(\mathrm{A}-\mathrm{H})$ Representative images of AFM of four LbL film configurations (condition 1, [CHT/HA-C/CHT/BG $]_{5}$-CHT/HA-C; condition 2, $[\mathrm{CHT} / \mathrm{HA}-\mathrm{C} / \mathrm{CHT} / \mathrm{BG}]_{5}$; control 1, [CHT/HA/CHT/BG $]_{5}$-CHT/HA; and control 2, $[\mathrm{CHT} / \mathrm{HA} / \mathrm{CHT} / \mathrm{BG}]_{5}$ ). (A-D) Phase contrast images; $(\mathrm{E}-\mathrm{H})$ height images. (I) Root-mean-square $\left(R_{\mathrm{q}}\right)$ and average roughness $\left(R_{\mathrm{a}}\right)$ of the studied surfaces $(n=3)$.

discussed. However, it should be noted that these estimated values of thickness assumes that the absorbed layer has a uniform thickness. Neto and co-workers ${ }^{3}$ obtained [CHT/HA$\mathrm{C}]_{5}$ films with thickness of approximately $75 \mathrm{~nm}$ and $[\mathrm{CHT} /$ $\mathrm{HA}]_{5}$ films (control) with around $130 \mathrm{~nm}$. Comparing these previous results with those obtained in this work for 10 layers from $[\mathrm{CHT} / \mathrm{HA}-\mathrm{C} / \mathrm{CHT} / \mathrm{BG}]_{6}$ and $[\mathrm{CHT} / \mathrm{HA} / \mathrm{CHT} / \mathrm{BG}]_{6}$ films ( 86 and $88 \mathrm{~nm}$, respectively), it was observed that these polymeric films have lower thickness than those obtained by Neto and co-workers. ${ }^{3}$ Moreover, in accordance with results presented by Neto and co-workers, ${ }^{3}$ multilayered films with dopamine $\left([\mathrm{CHT} / \mathrm{HA}-\mathrm{C} / \mathrm{CHT} / \mathrm{BG}]_{6}\right)$ were thinner than the control films, suggesting that the combination of dopamine with bioactive nanoparticles should be responsible to make these multilayered films more compact.

Therefore, since the sequential depositions were stable and reproducible in the several QCM-D experiments performed, it was shown that multifunctional films containing both bioactive nanoparticles and dopamine could be successful assembled using the LbL technique.

Production and Characterization of Multifunctional LbL Coatings. Distinct films were produced as illustrated in Figure 3: multifunctional 10-bilayered films containing CHT, $\mathrm{HA}-\mathrm{C}$, and BG ending either with a HA-C layer (Figure 3C) or with a BG layer (Figure 3D); control 10-bilayered films containing CHT, HA, and BG (Figure 3F,G), that is, films without dopamine; and multifunctional 11-bilayered films (Figure 3B) and respective control (Figure 3E) ending with a HA-C/HA layer. Multifunctional and control films produced onto glass substrates were then structurally and mechanically and characterized.

The topography and surface roughness of these multilayered films were analyzed by atomic force microscopy (AFM). Figure $4 \mathrm{~A}-\mathrm{H}$ shows surfaces with a nanoscale roughness provided by a heterogeneous agglomeration of BG nanoparticles on the surface of the films, in accordance with findings of Couto and co-workers. ${ }^{19,38}$ Based on the average roughness $\left(R_{a}\right)$ and the root-mean-square $\left(R_{\mathrm{q}}\right)$ values of each deposited thin films (Figure 4I), it appears that all surfaces presented a similar nanoscale roughness, since the $R_{\mathrm{a}}$ and $R_{\mathrm{q}}$ values are not very distant. Moreover, there are no differences in the roughness values determined for coatings with and without dopamine. It was shown in a previous work $^{3}$ that $[\mathrm{CHT} / \mathrm{HA}-\mathrm{C}]_{10}$ films presented a rougher surface when compared with the control film, $[\mathrm{CHT} / \mathrm{HA}]_{10}$. However, in the present work, there are no differences in the surface roughness values, probably due to the effect of BG nanoparticle agglomeration that was incorporated in all LbL coatings. In this sense, it was suggested that such organization maintains the inorganic component strongly bonded to the polymeric component of LbL films, because polymers are deposited and inserted between nanoparticles and they act as a glue. ${ }^{3}$

For the adhesion experiments, pairs of samples with different LbL film configurations were bonded and an axial tensile force was applied at a constant cross-head speed until the detachment of the glass plaques. Figure 5 presents the mean values of adhesive strength obtained from five lap shear tests performed for each LbL film configuration, which indicates significant differences in the adhesive strength of the films. In particular, the adhesive strength of the multifunctional films is significantly higher when compared with the respective control: [CHT/HA$\mathrm{C} / \mathrm{CHT} / \mathrm{BG}]_{5}+\mathrm{CHT} / \mathrm{HA}-\mathrm{C}$ films present an adhesive strength of $2.09 \pm 0.04 \mathrm{MPa}$, whereas the value for their control, [CHT/ $\mathrm{HA} / \mathrm{CHT} / \mathrm{BG}]_{5}+\mathrm{CHT} / \mathrm{HA}$, is $0.86 \pm 0.08 \mathrm{MPa}$; $[\mathrm{CHT} / \mathrm{HA}-$ $\mathrm{C} / \mathrm{CHT} / \mathrm{BG}]_{5}$ films present an adhesive strength of $1.16 \pm$ $0.04 \mathrm{MPa}$, and their control value, $[\mathrm{CHT} / \mathrm{HA} / \mathrm{CHT} / \mathrm{BG}]_{5}$, is $0.79 \pm 0.04 \mathrm{MPa}$. In fact, $\mathrm{Yu}$ and co-workers have shown that dopamine forms moisture-resistant adhesive bonds with several substrates, and reported adhesive strength values between 0.56 $\pm 0.18 \mathrm{MPa}$ and $4.70 \pm 0.87 \mathrm{MPa}$, depending on the substrate 


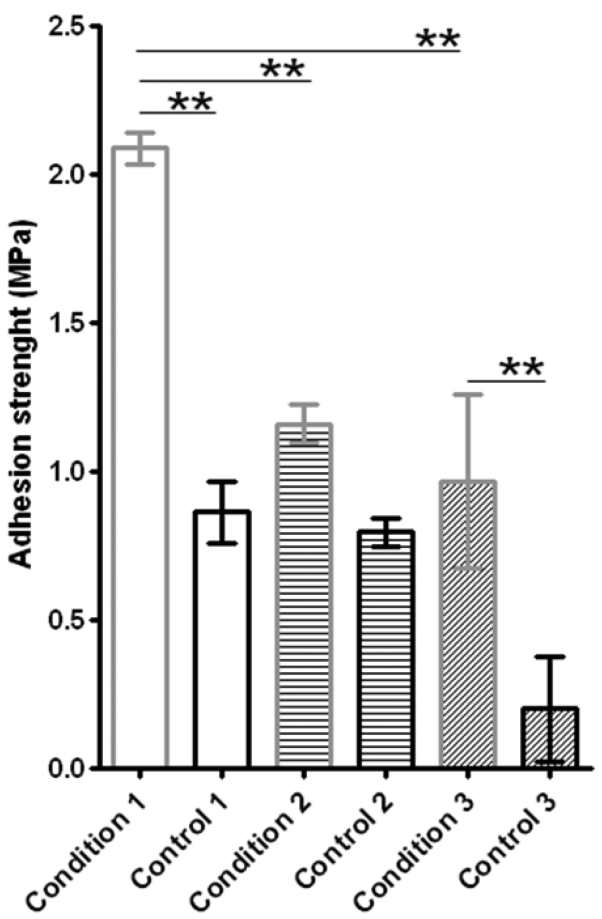

Figure 5. Adhesive strength of four LbL film conditions (condition 1, [CHT/HA-C/CHT/BG $]_{5}$-CHT/HA-C; condition 2, [CHT/HA-C/ $\mathrm{CHT} / \mathrm{BG}]_{5}$; condition 3, [CHT/HA-C $]_{10}$; control 1, [CHT/HA/ $\mathrm{CHT} / \mathrm{BG}]_{5}-\mathrm{CHT} / \mathrm{HA}$; control 2, $[\mathrm{CHT} / \mathrm{HA} / \mathrm{CHT} / \mathrm{BG}]_{5}$; and control 3, $\left.[\mathrm{CHT} / \mathrm{HA}]_{10}\right)$ between glass slides. Data are means \pm standard deviation $(n=5 ; * *<0.01)$.

and oxidation conditions. ${ }^{39}$ According to the findings of Neto and co-workers, ${ }^{3}$ multifunctional films containing hyaluronic acid modified with dopamine evidence enhanced adhesive properties, even with or without nanoparticles in their constitution. However, the adhesive strength of multifunctional films ending with the adhesive layer and the one of the multifunctional films ending with the bioactive layer is statistically different ( $p$-value $<0.01)$, where [CHT/HA-C/ $\mathrm{CHT} / \mathrm{BG}]_{5}+\mathrm{CHT} / \mathrm{HA}-\mathrm{C}$ films show a higher adhesive strength than $[\mathrm{CHT} / \mathrm{HA}-\mathrm{C} / \mathrm{CHT} / \mathrm{BG}]_{5}$ films. This could be due to the enhanced adhesive properties that HA-C provides as the end layer of these multifunctional films.

In Vitro Bioactivity Studies. In vitro bioactivity tests were conducted in order to investigate the apatite formation under physiological-like conditions onto the films developed with distinct configurations. Figure $6 \mathrm{~A}-\mathrm{H}$ shows the scanning electron microscopy (SEM) images, before (0 days) and after (7 days) immersion in SBF solution. Before the SBF immersion (0 days), it is possible to observe heterogeneous nanoparticle agglomerations on the film surface for all the film configurations. For $[\mathrm{CHT} / \mathrm{HA}-\mathrm{C} / \mathrm{CHT} / \mathrm{BG}]_{5}$ and $[\mathrm{CHT} /$ $\mathrm{HA} / \mathrm{CHT} / \mathrm{BG}]_{5}$, the energy dispersive X-ray spectroscoy (EDS) analysis confirmed the presence of the bioactive glass nanoparticles used $\left(\mathrm{SiO}_{2} / \mathrm{CaO} / \mathrm{P}_{2} \mathrm{O}_{5}(\mathrm{~mol} \%)=50: 45: 5\right)$ and the presence of carbon $(\mathrm{C})$, oxygen $(\mathrm{O})$, sodium $(\mathrm{Na})$, high amounts of silicon $(\mathrm{Si})$, vestiges of magnesium $(\mathrm{Mg})$, aluminum (Al), and calcium $(\mathrm{Ca})$. For $[\mathrm{CHT} / \mathrm{HA}-\mathrm{C}]_{10}$ and $[\mathrm{CHT} / \mathrm{HA}]_{10}$, the SEM images reveal a smoother surface in comparison with the previous LbL configurations, and the EDS spectra presented in SFigure 1 (Supporting Information) show elements $(\mathrm{C}$ and $\mathrm{O})$ from organic materials and also residues from the glass substrate. All films reveal the presence of magnesium and aluminum, probably due to the glass substrate used.

After immersion (7 days), the SEM images evidenced that the surface of coatings presents apatite-like structures with the typical cauliflower morphology, already observed in a previous work. $^{28}$ Accordingly with the peaks evolution mechanism explained by Luz and Mano, ${ }^{28}$ the EDS analysis reveals lower $\mathrm{Si}$
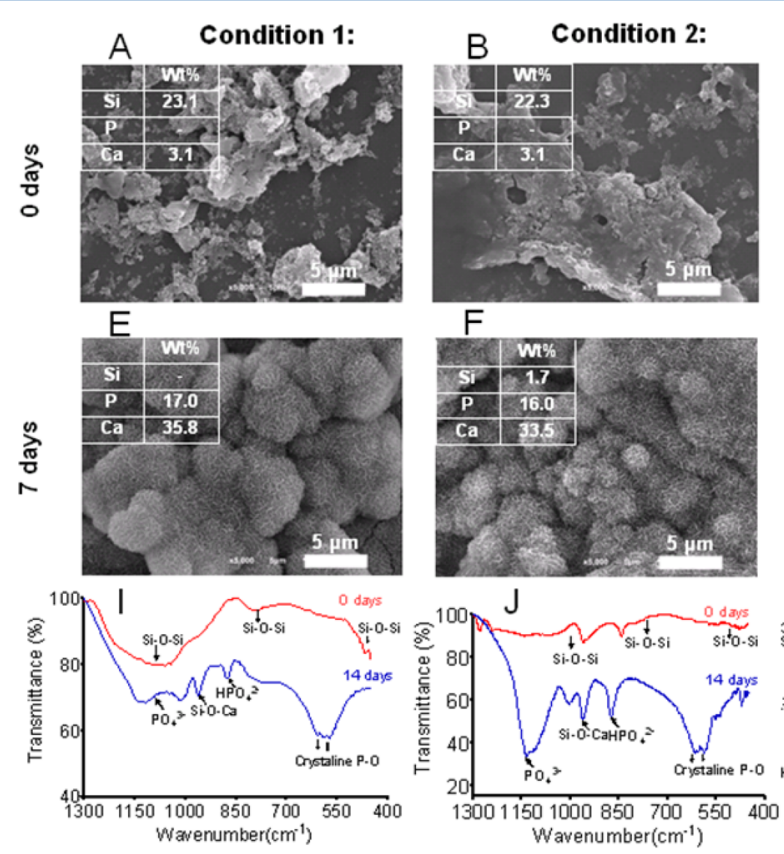
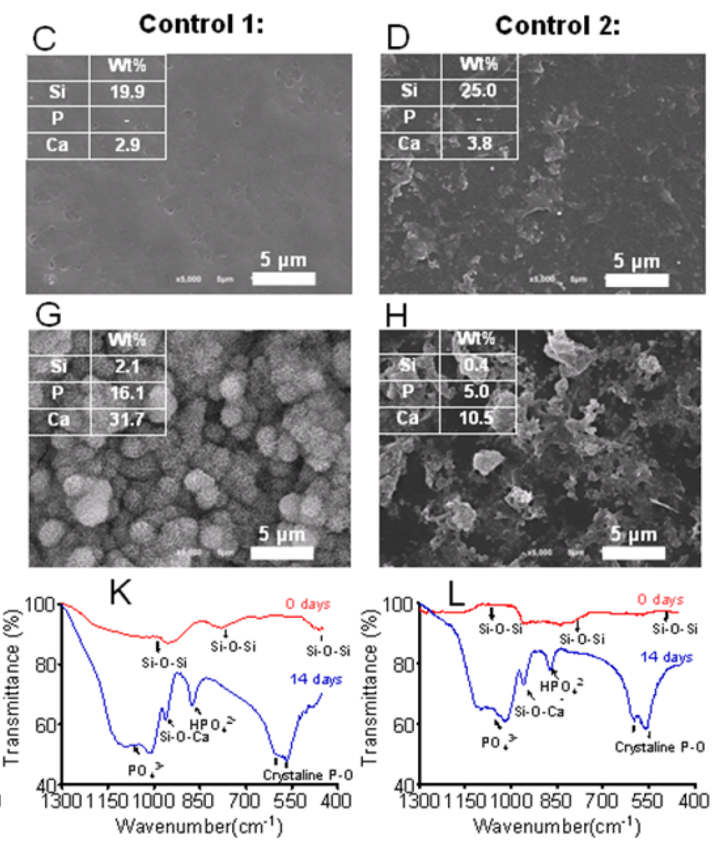

Figure 6. In vitro bioactivity studies. (A-H) Representative images of scanning electron microscopy with quantitative EDS analysis of four LbL film configurations (condition 1, [CHT/HA-C/CHT/BG $]_{5}-\mathrm{CHT} / \mathrm{HA}-\mathrm{C}$; condition 2, $[\mathrm{CHT} / \mathrm{HA}-\mathrm{C} / \mathrm{CHT} / \mathrm{BG}]_{5}$; control 1, $[\mathrm{CHT} / \mathrm{HA} / \mathrm{CHT} / \mathrm{BG}]_{5}-$ $\mathrm{CHT} / \mathrm{HA}$; and control 2, [CHT/HA/CHT/BG $\left.]_{5}\right)(\mathrm{A}-\mathrm{D})$ before and $(\mathrm{E}-\mathrm{H})$ after the immersion in SBF for 7 days. (I-L) Corresponding FTIR spectra obtained before and after the immersion in SBF for 14 days. 
peaks, and the presence of $\mathrm{Ca}$ and $\mathrm{P}$ peaks, which tend to increase during the SBF immersion due to the nanoparticles dissolution, which provides the formation of an apatite layer. These results confirm the bioactive behavior of all LbL configurations tested, which promoted the development of the typical cauliflower morphology of apatite crystals.

Figure 6I-L presents the Fourier transform infrared (FTIR) spectrum of the film surface before ( 0 days $)$ and after immersion (14 days) in SBF. Before the SBF immersion, it is possible to recognize the silicate absorption bands: $1085 \mathrm{~cm}^{-1}$ (asymmetric stretching mode), $800 \mathrm{~cm}^{-1}$ (symmetric stretching vibration), and $464 \mathrm{~cm}^{-1}$ (rocking vibration of $\mathrm{Si}-\mathrm{O}-\mathrm{S}$ ). As it was mentioned previously, these bands tend to disappear due to the decrease of Si content after SBF immersion. ${ }^{28}$ After SBF immersion, all spectra reveal the characteristic peaks of carbonated apatite. The main evidence of hydroxyapatite growth is the presence of bands of the $\mathrm{P}-\mathrm{O}$ bending vibration due to the crystalline calcium phosphate phase (600 to 550 $\left.\mathrm{cm}^{-1}\right)$, the band of acidic phosphate group, $\mathrm{HPO}_{4}{ }^{2-}(874$ $\mathrm{cm}^{-1}$ ), the peaks assigned to the Ca presence associated with $\mathrm{Si}-\mathrm{O}-\mathrm{Ca}$ bonds $\left(950 \mathrm{~cm}^{-1}\right)$, and the bands representative of the stretching mode of $\mathrm{PO}_{4}^{-3}$ group $\left(1045-1200 \mathrm{~cm}^{-1}\right){ }^{28}$ So, it is possible to conclude that, after 7 days of SBF immersion, the FTIR spectrum exhibited the typical absorption peaks of hydroxyapatite, in agreement with the SEM and EDS analysis, confirming the development of a carbonated apatite mineral. As it was expected, $[\mathrm{CHT} / \mathrm{HA}-\mathrm{C}]_{10}$ films do not present those apatite bands.

Figure 7 shows the X-ray diffraction (XRD) characterization for multilayered films with and without nanoparticles, before $(0$

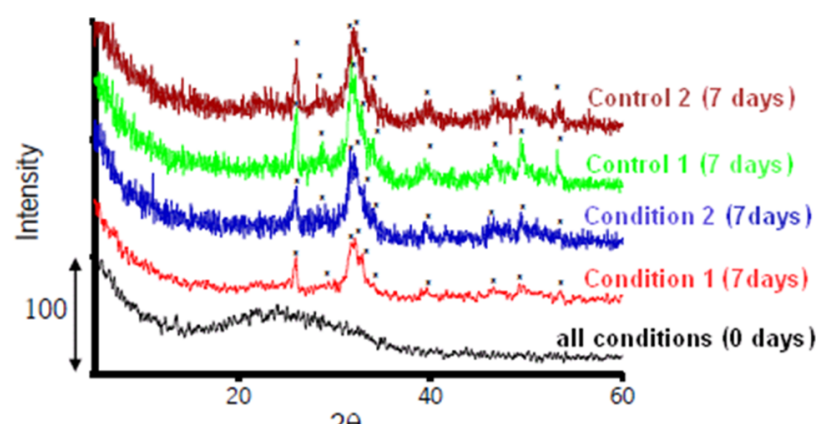

$2 \theta$

Figure 7. XRD diffractograms obtained for each LbL film configuration before and after the immersion in SBF for 7 days. *Principal characteristic hydroxyapatite peaks are indicated.

days) and after immersion in SBF solution (7 days). The XRD spectrum before the immersion in SBF shows an amorphous profile due to the glass substrate used, which does not have maximum detectable diffraction. After immersion in SBF, there are two well-defined intense peaks attributed as the characteristic crystalline diffraction pattern of hydroxyapatite at $2 \theta=26^{\circ}$ [(111) diffraction peak] and $2 \theta=32^{\circ}$ [overlapping of (211), (112), and (300) diffraction peaks]. ${ }^{28}$ Besides these two peaks, there are also other characteristic hydroxyapatite peaks with less intensity at $2 \theta=29^{\circ}, 31.8^{\circ}, 32.9^{\circ} .34 .7^{\circ}, 39.8^{\circ}, 46.7^{\circ}, 49.7^{\circ}$, and $53.1^{\circ}$, with lattice dimensions of $a=b=9.4817 \AA$ and $c=$ $6.8843 \AA$, as shown on the marks in the Figure 7. The peaks with small intensity are attributed to the partial crystalline structure, while their broadness is due to the small crystalline structures, comparable to the ones present in bone. One of the reasons that can explain the apatite peaks' presence is the precipitation of hydroxyapatite from the amorphous structure of silica-based bioactive glasses in the aqueous medium of the sol-gel process. ${ }^{25}$

The XRD pattern of multilayered films appears to have the typical hydroxyapatite diffractrogram; moreover, the obtained results were similar to the one reported by previous works, ${ }^{19,28}$ indicating that these results prove once again that the multifunctional films produced in this study are bioactive and could be potentially used for orthopedic applications. As expected, the multilayered films without nanoparticles do not present those hydroxyapatite characteristic peaks.

In Vitro Compatibility Tests. Direct contact tests were carried out in order to study the interactions between the cells and multilayered films. The results are depicted in Figure 8.

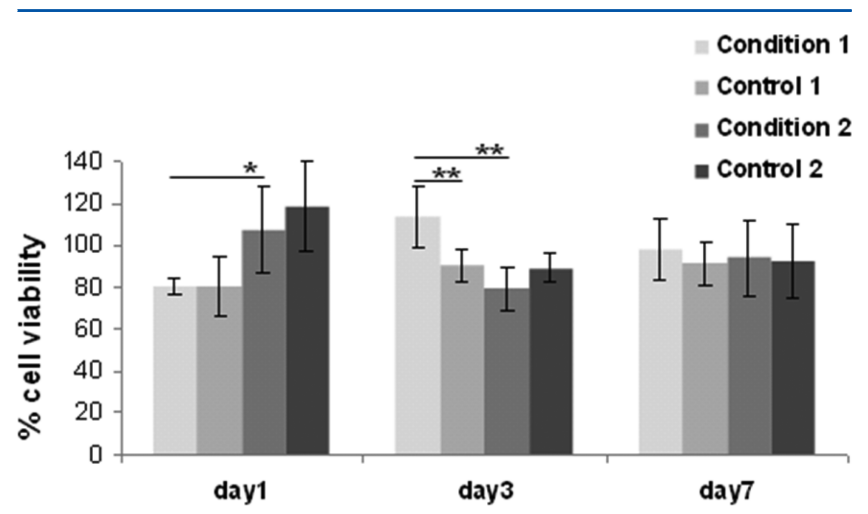

Figure 8. In vitro biocompatibility tests. The cellular viability of four LbL film configurations (condition 1 and 2, and control 1 and 2) tested for L929 cells was determined using the MTS assay. All results are represented as mean \pm standard deviation. Statistical differences in grouped by time point analysis are marked with $(*)$ and $(* *)$, which stand for $p$-value $<0.05$ and $p$-value $<0.001$, respectively.

The cellular viability after seeding on the surface of the developed films was determined using the MTS assay. The response of the four LbL film configurations depicted in Figure 3 was evaluated in this study: condition 1 (Figure 3A), condition 2 (Figure 3B), control 1 (Figure 3D), and control 2 (Figure $3 \mathrm{E}$ ). It can be seen that the cellular viability in the presence of the films is above $80 \%$, so one may conclude that these multifunctional films are nontoxic toward the cells metabolism, assuring the materials biocompatibility.

Comparing conditiosn 1 and 2 with their controls, no obvious influence of the presence of the dopamine layer is sensed by the cells over the three time points. In the first day, condition 1 presents significant difference compared to condition 2, and, as expected, both condition 2 and its control indicate higher cellular viability in comparison with the first condition and its control, due to the presence of the BG layer, whose ion leachables are known to have a good influence on cellular metabolism. However, after 3 days, condition 1 presents higher viability than condition 2 , and this significant difference should be related with the excess of BG dissolution that could increase the $\mathrm{pH}$ of the culture medium, decreasing the cell viability for condition 2 . Surprisingly, on day 3, condition 1 has cell viability significant higher than its control, but after this time point, all conditions stabilize in similar values, probably due to the excess of proliferation in the limited space of the well. 


\section{CONCLUSIONS}

Nanostructured coatings inspired in the composition of nacre, combining organic layers of chitosan, hyaluronic acid, or dopamine-modified hyaluronic acid with inorganic layers constituted by bioactive glass nanoparticles, were successfully developed under the scope of this work. The results proved that these innovative multilayered polymeric coatings are nontoxic and that they combine enhanced adhesive properties with a bioactive character. Such films could be applied in the orthopedic field, in particular, as coatings of orthopedic implants able to form a biocompatible and adhesive interface with bone.

\section{ASSOCIATED CONTENT}

\section{S Supporting Information}

The Supporting Information is available free of charge on the ACS Publications website at DOI: 10.1021/acs.langmuir.5b03508.

SFigure 1 complementing the EDS analysis presented in Figure $6 \mathrm{~A}-\mathrm{H}(\mathrm{PDF})$

\section{AUTHOR INFORMATION}

\section{Corresponding Author}

*E-mail: nalves@dep.uminho.pt.

\section{Author Contributions}

${ }^{\S}$ S.J.R. and A.C.V. contributed equally to this work.

\section{Notes}

The authors declare no competing financial interest.

\section{ACKNOWLEDGMENTS}

The authors want to acknowledge the Portuguese Foundation for Science and Technology (FCT) and the European program FEDER/COMPETE for the financial support through project BioSeaGlue: EXPL/CTM-BIO/0646/2013 (FCOMP-01-0124FEDER-041105).

\section{REFERENCES}

(1) Podsiadlo, P.; Paternel, S.; Rouillard, J. M.; Zhang, Z.; Lee, J.; Lee, J. W.; Gulari, E.; Kotov, N. A. Layer-by-layer assembly of nacrelike nanostructured composites with antimicrobial properties. Langmuir 2005, 21 (25), 11915-21.

(2) Yamada, K.; Chen, T.; Kumar, G.; Vesnovsky, O.; Topoleski, L. D.; Payne, G. F. Chitosan based water-resistant adhesive. Analogy to mussel glue. Biomacromolecules 2000, 1 (2), 252-8.

(3) Neto, A. I.; Cibrao, A. C.; Correia, C. R.; Carvalho, R. R.; Luz, G. M.; Ferrer, G. G.; Botelho, G.; Picart, C.; Alves, N. M.; Mano, J. F. Nanostructured polymeric coatings based on chitosan and dopaminemodified hyaluronic acid for biomedical applications. Small 2014, 10 (12), 2459-69.

(4) Deming, T. J. Mussel byssus and biomolecular materials. Curr. Opin. Chem. Biol. 1999, 3 (1), 100-5.

(5) Lee, H.; Lee, Y.; Statz, A. R.; Rho, J.; Park, T. G.; Messersmith, P. B. Substrate-Independent Layer-by-Layer Assembly by Using MusselAdhesive-Inspired Polymers. Adv. Mater. 2008, 20 (9), 1619-23.

(6) Neto, A. I.; Meredith, H. J.; Jenkins, C. L.; Wilker, J. J.; Mano, J. F. Combining biomimetic principles from the lotus leaf and mussel adhesive: polystyrene films with superhydrophobic and adhesive layers. RSC Adv. 2013, 3 (24), 9352-9356.

(7) Nicklisch, S. C. T.; Waite, J. H. Mini-review: The role of redox in Dopa-mediated marine adhesion. Biofouling 2012, 28 (8), 865-877.

(8) Lee, Y.; Lee, H.; Kim, Y. B.; Kim, J.; Hyeon, T.; Park, H.; Messersmith, P. B.; Park, T. G. Bioinspired Surface Immobilization of Hyaluronic Acid on Monodisperse Magnetite Nanocrystals for Targeted Cancer Imaging. Adv. Mater. 2008, 20 (21), 4154-4157.
(9) Charlot, A.; Sciannamea, V.; Lenoir, S.; Faure, E.; Jerome, R.; Jerome, C.; Van De Weerdt, C.; Martial, J.; Archambeau, C.; Willet, N.; Duwez, A.-S.; Fustin, C.-A.; Detrembleur, C. All-in-one strategy for the fabrication of antimicrobial biomimetic films on stainless steel. $J$. Mater. Chem. 2009, 19 (24), 4117-4125.

(10) Guvendiren, M.; Messersmith, P. B.; Shull, K. R. Self-Assembly and Adhesion of DOPA-Modified Methacrylic Triblock Hydrogels. Biomacromolecules 2008, 9 (1), 122-128.

(11) Ku, S. H.; Ryu, J.; Hong, S. K.; Lee, H.; Park, C. B. General functionalization route for cell adhesion on non-wetting surfaces. Biomaterials 2010, 31 (9), 2535-41.

(12) Lee, H.; Scherer, N. F.; Messersmith, P. B. Single-molecule mechanics of mussel adhesion. Proc. Natl. Acad. Sci. U. S. A. 2006, 103 (35), 12999-3003.

(13) Nicklisch, S. C.; Waite, J. H. Mini-review: the role of redox in Dopa-mediated marine adhesion. Biofouling 2012, 28 (8), 865-77.

(14) Sever, M. J.; Weisser, J. T.; Monahan, J.; Srinivasan, S.; Wilker, J. $\mathrm{J}$. Metal-mediated cross-linking in the generation of a marine-mussel adhesive. Angew. Chem., Int. Ed. 2004, 43 (4), 448-50.

(15) Luz, G. M.; Mano, J. F. Mineralized structures in nature: Examples and inspirations for the design of new composite materials and biomaterials. Compos. Sci. Technol. 2010, 70 (13), 1777-1788.

(16) Barthelat, F. Designing nacre-like materials for simultaneous stiffness, strength and toughness: Optimum materials, composition, microstructure and size. J. Mech. Phys. Solids 2014, 73, 22-37.

(17) Chintapalli, R. K.; Breton, S.; Dastjerdi, A. K.; Barthelat, F. Strain rate hardening: a hidden but critical mechanism for biological composites? Acta Biomater. 2014, 10 (12), 5064-73.

(18) Li, X.; Xu, Z.-H.; Wang, R. In Situ Observation of Nanograin Rotation and Deformation in Nacre. Nano Lett. 2006, 6 (10), 23012304.

(19) Couto, D. S.; Alves, N. M.; Mano, J. F. Nanostructured multilayer coatings combining chitosan with bioactive glass nanoparticles. J. Nanosci. Nanotechnol. 2009, 9 (3), 1741-8.

(20) Tang, Z.; Wang, Y.; Podsiadlo, P.; Kotov, N. A. Biomedical Applications of Layer-by-Layer Assembly: From Biomimetics to Tissue Engineering. Adv. Mater. 2006, 18 (24), 3203-3224.

(21) Burghard, Z.; Tucic, A.; Jeurgens, L. P. H.; Hoffmann, R. C.; Bill, J.; Aldinger, F. Nanomechanical Properties of Bioinspired Organic-Inorganic Composite Films. Adv. Mater. 2007, 19 (7), 970-974.

(22) Podsiadlo, P.; Liu, Z.; Paterson, D.; Messersmith, P. B.; Kotov, N. A. Fusion of Seashell Nacre and Marine Bioadhesive Analogs: HighStrength Nanocomposite by Layer-by-Layer Assembly of Clay and L3,4-Dihydroxyphenylalanine Polymer. Adv. Mater. 2007, 19 (7), 949955.

(23) Hench, L. L. The story of Bioglass. J. Mater. Sci.: Mater. Med. 2006, 17 (11), 967-78.

(24) Alves, N. M.; Leonor, I. B.; Azevedo, H. S.; Reis, R. L.; Mano, J. F. Designing biomaterials based on biomineralization of bone. J. Mater. Chem. 2010, 20 (15), 2911-2921.

(25) Hong, Z.; Reis, R. L.; Mano, J. F. Preparation and in vitro characterization of novel bioactive glass ceramic nanoparticles. $J$. Biomed. Mater. Res., Part A 2009, 88 (2), 304-313.

(26) Hong, Z.; Reis, R. L.; Mano, J. F. Preparation and in vitro characterization of scaffolds of poly(L-lactic acid) containing bioactive glass ceramic nanoparticles. Acta Biomater. 2008, 4 (5), 1297-306.

(27) Gerhardt, L. C.; Widdows, K. L.; Erol, M. M.; Burch, C. W.; Sanz-Herrera, J. A.; Ochoa, I.; Stampfli, R.; Roqan, I. S.; Gabe, S.; Ansari, T.; Boccaccini, A. R. The pro-angiogenic properties of multifunctional bioactive glass composite scaffolds. Biomaterials 2011, 32 (17), 4096-108.

(28) Luz, G. M.; Mano, J. F. Preparation and characterization of bioactive glass nanoparticles prepared by sol-gel for biomedical applications. Nanotechnology 2011, 22 (49), 494014.

(29) Luz, G. M.; Mano, J. F. Biomimetic design of materials and biomaterials inspired by the structure of nacre. Philos. Trans. R. Soc., A 2009, 367 (1893), 1587-605. 
(30) Lee, Y.; Chung, H. J.; Yeo, S.; Ahn, C.-H.; Lee, H.; Messersmith, P. B.; Park, T. G. Thermo-sensitive, injectable, and tissue adhesive solgel transition hyaluronic acid/pluronic composite hydrogels prepared from bio-inspired catechol-thiol reaction. Soft Matter 2010, 6 (5), 977-983.

(31) Alves, N. M.; Picart, C.; Mano, J. F. Self assembling and crosslinking of polyelectrolyte multilayer films of chitosan and alginate studied by QCM and IR spectroscopy. Macromol. Biosci. 2009, 9 (8), 776-85.

(32) Martins, G. V.; Merino, E. G.; Mano, J. F.; Alves, N. M. Crosslink effect and albumin adsorption onto chitosan/alginate multilayered systems: an in situ QCM-D study. Macromol. Biosci. 2010, 10 (12), 1444-55.

(33) Kokubo, T.; Takadama, H. How useful is SBF in predicting in vivo bone bioactivity? Biomaterials 2006, 27 (15), 2907-2915.

(34) Hong, S.; Yang, K.; Kang, B.; Lee, C.; Song, I. T.; Byun, E.; Park, K. I.; Cho, S.-W.; Lee, H. Hyaluronic Acid Catechol: A Biopolymer Exhibiting a pH-Dependent Adhesive or Cohesive Property for Human Neural Stem Cell Engineering. Adv. Funct. Mater. 2013, 23 (14), 1774-1780.

(35) Wu, J.; Zhang, L.; Wang, Y.; Long, Y.; Gao, H.; Zhang, X.; Zhao, N.; Cai, Y.; Xu, J. Mussel-Inspired Chemistry for Robust and SurfaceModifiable Multilayer Films. Langmuir 2011, 27 (22), 13684-13691.

(36) Li, X. Nanoscale structural and mechanical characterization of natural nanocomposites: Seashells. JOM 2007, 59 (3), 71-74.

(37) Hook, F.; Kasemo, B.; Nylander, T.; Fant, C.; Sott, K.; Elwing, $\mathrm{H}$. Variations in coupled water, viscoelastic properties, and film thickness of a Mefp-1 protein film during adsorption and cross-linking: a quartz crystal microbalance with dissipation monitoring, ellipsometry, and surface plasmon resonance study. Anal. Chem. 2001, 73 (24), 5796-804.

(38) Couto, D. S.; Hong, Z.; Mano, J. F. Development of bioactive and biodegradable chitosan-based injectable systems containing bioactive glass nanoparticles. Acta Biomater. 2009, 5 (1), 115-123.

(39) Yu, M.; Deming, T. J. Synthetic Polypeptide Mimics of Marine Adhesives. Macromolecules 1998, 31 (15), 4739-4745. 